\title{
A hybrid water cycle particle swarm optimization for solving the fuzzy underground water confined steady flow
}

\author{
E. M. Badr ${ }^{1}$, H. S. AlGendy ${ }^{2}$ \\ ${ }^{1}$ Department of scientific computing, Faculty of computer and informatics, Benha University, Egypt \\ ${ }^{1}$ Higher Technological Institute, Egypt \\ ${ }^{2}$ Department of Basic Engineering Science, Benha Faculty of Engineering, Benha University, Egypt
}

\begin{tabular}{l} 
Article Info \\
\hline Article history: \\
Received Jul 21, 2019 \\
Revised Dec 21, 2019 \\
Accepted Feb 18, 2020 \\
\hline
\end{tabular}

Keywords:

Benchmark problems

Confined steady underground

flow

Fuzzy multiobjective

programming

Hybrid optimization

Particle swarm algorithm

Water cycle algorithm

\begin{abstract}
Groundwater sustainability is the development and use of groundwater resources to meet current and future beneficial uses without causing unacceptable environmental or socioeconomic consequences. This study is the first time to apply the hybrid optimization technique for solving of managing underground water aquifers, the confined steady flow problems, where a hybrid water cycle - particle swarm optimization WCA-PSO is proposed. In particular, we introduce a novel hybrid algorithm using water cycle algorithm (WCA) and particle swarm Optimization (PSO). The performance of the novel hybrid algorithm WCA-PSO is evaluated to solve 10 benchmark problems chosen from literature. The simulation results and comparison with pure WCA and PSO algorithms confirm the effectiveness of the proposed algorithm WCA-PSO for solving various benchmark optimization functions. Finally, we solve the problem of managing underground water aquifers by WCA, PSO and the hybrid optimization WCA-PSO. The experimental results analysis and statistical tests prove that the hybrid algorithm WCA-PSO overcomes the pure algorithms.
\end{abstract}

Copyright $(9) 2020$ Institute of Advanced Engineering and Science. All rights reserved.

\section{Corresponding Author:}

Elsayed Badr,

Department of scientific computing,

Faculty of computer and informatics,

Benha University, Egypt.

Email: badrgraph@ gmail.com

\section{INTRODUCTION}

Many real-world optimization problems are very complex and challenging to solve, and many applications have to deal with these problems. To solve such problems, approximate optimization methods have to be used, though there is no guarantee that the optimal solution can be obtained [1]. Nature has been solving many problems for billions of years, and many kinds of biological systems have shown fascinating and remarkable efficiency in problem solving [2-4]. Over the last few decades optimization algorithms have been applied in extensive numbers of difficult problems. Several nature-inspired algorithms have been developed over the last few years by the scientific community [2-5].

Water management is a major challenge facing the different countries due to water increasing needs in all fields of life. More attention has been devoted to understanding and managing the transition from current management regimes to more adaptive regimes. So, we will manage underground water aquifers where it based on the finite difference approximation to the system as which treated through fuzziness environment. The uncertainty due to imprecise data may be come from indirect measurements, expert judgment, or subjective to the interpretation of available information. Also, the finite difference method is used to approximate the governing equation of groundwater flow, in which aquifer parameters such as transmissivity are to be considered as a fuzzy number. So, the variables in the system are fuzzy instead of its 
crisp values and then the dependent variable (e.g. hydraulic head) is also fuzzy. When the transmissivity is represented as a fuzzy number, the membership function of the hydraulic head outputs can be easily determined based on the analytical solution. At each level, both the transmissivity and hydraulic heads are transformed into intervals. Since there are not research studies which use the hybrid optimization technique for solving this problem. So, this work is the first time to apply the hybrid optimization technique for solving of managing underground water aquifers, the confined steady flow problems, where a hybrid water cycle particle swarm optimization WCA-PSO is proposed.

Particle swarm optimization (PSO) algorithm is nature-inspired population-based metaheuristic algorithms originally accredited to Eberhart, Kennedy, and Russell Eberhart in 1995 [6]. This algorithm mimics the social behavior of birds flocking and fishes schooling. Starting form a randomly distributed set of particles (potential solutions), the algorithm try to improve the solutions according to a quality measure (fitness function). The improvisation is performed through moving the particles around the search space by means of a set of simple mathematical expressions which model some interparticle communications [7].

The water cycle process, also known as the hydrological or the $\mathrm{H}_{2} \mathrm{O}$ cycle, explains the unceasing movement of water on, above, and below the surface of the earth. As we observe in nature, streams flow into rivers and rivers flow into the sea. Finally, all the rivers and/or streams end up in the sea, the most downhill (low-altitude) place in the world [8]. Therefore, similar to a metaheuristic swarm optimization algorithm, this phenomenon lends itself to finding a global optimal solution or a near-optimal solution via effective exploration and exploitation. Inspired by this observation, the water cycle algorithm (WCA) has been developed as a new metaheuristic algorithm [9].

In this work, we introduce a novel hybrid algorithm using water cycle algorithm (WCA) and particle swarm Optimization (PSO). The performance of the novel hybrid algorithm WCA-PSO is evaluated to solve 10 benchmark problems chosen from literature. The simulation results and comparison with pure WCA and PSO algorithms confirm the effectiveness of the proposed algorithm WCA-PSO for solving various benchmark optimization functions. Finally, we solve the problem of managing underground water aquifers by WCA, PSO and the hybrid optimization WCA-PSO. The experimental results analysis and statistical tests prove that the hybrid algorithm WCA-PSO overcomes the other algorithms.

The remaining of this paper is organized as follows: particle swarm optimization details and its procedure are described in Section 2. In Section 3, detailed descriptions of the water cycle algorithm (WCA) and their concepts are introduced. The proposed algorithm is discussed in Section 4. Benchmark functions accompanied with their mathematical formulations considered in this paper and the comparisons of the obtained statistical optimization results using the WCA-PSO with other traditional optimization algorithms PSO, WCA for reported problems in form of tables and figures are provided in Section 5. Section 6 describes the multiobjective fuzzy optimization model for aquifer management. Section 7 provides details of the solution and analysis results model for the aquifer management problem, also parameter settings of the algorithms and compares their results. Finally, conclusions are drawn in Section 8.

\section{PARTICLE SWARM ALGORITHM}

Particle swarm optimization (PSO) algorithm is nature-inspired population-based metaheuristic algorithms mimic the social behavior of birds flocking and fishes schooling [6, 7]. It is considered a stochastic optimization approach based on population search. These algorithms individuals, referred to as particles, are grouped into a swarm, and each particle in the swarm represents a feasible solution to the problem in the search space. The performance of each particle is measured according to a predefined fitness function which is related to the problem being solved [10]. PSO use a population of individual particles where each particle has a position, a velocity, and memory of the location of its best fitness found during the search process. Each particle updates its velocity and memory, and then the memory of other particles is shared in its neighborhood. By updating the velocity, the particle will move to a new position in the search. The main steps of the cuckoo search algorithm are summarized in Algorithm 1. The PSO in its original form is defined by $[11,12]$ :

$$
\begin{aligned}
& V_{i d}^{t+1}=w \cdot v_{i d}^{t}+c_{1} \cdot r_{1 d}^{t}\left(P_{b e s t, i}^{t}-x_{i d}^{t}\right)+c_{2} \cdot r_{2 d}^{t}\left(G_{b e s t}^{t}-x_{i d}^{t}\right) \\
& X_{i d}^{t+1}=X_{i d}^{t}+V_{i d}^{t+1}, \quad d=1,2, \ldots . n
\end{aligned}
$$

where $v_{i d}^{t}$ and $x_{i d}^{t} \quad$ the velocity and position vectors of particle $i$ in dimension $d$ at time $t$, respectively, $w$ is representative of the inertia weight, $P_{\text {best }, i}^{t}$ is the personal best position of particle $i, G_{\text {best }}^{t}$ is the global best position of particle $i, c_{1}, c_{2}$ are positive acceleration constants which are used to level the contribution of the cognitive and social components respectively; $r_{1 d}^{t}, r_{2 d}^{t}$ are random numbers from uniform distribution $\mathrm{U}(0,1)$ at time. 


\section{WATER CYCLE ALGORITHM}

The WCA mimics the flow of rivers and streams toward the sea and was derived by observing the water cycle process. Assume that there are some rain or precipitation phenomena. An initial population of design variables is randomly generated after the raining process. The best individual, classified in terms of having the minimum cost function (for minimization problems), is chosen as the sea [13].

Then, a number of good streams are chosen as rivers, whereas the remaining streams flow into the rivers and the sea. Starting the optimization algorithm requires the generation of an initial population representing a matrix of streams of size $N p o p \times D$, where $\mathrm{D}$ is the dimension and $(N p o p)$ is the population size. Hence, this matrix, which is generated randomly, is given as:

$$
\text { Total population }=\left[\begin{array}{c}
\text { sea } \\
\text { River }_{1} \\
\text { River }_{2} \\
\vdots \\
\text { Stream }_{N s r+1} \\
\text { Stream }_{N s r+2} \\
\text { Stream }_{N s r+3} \\
\vdots \\
\text { Stream }_{N p o p}
\end{array}\right]=\left[\begin{array}{ccccc}
x_{1}^{1} & x_{2}^{1} & x_{3}^{1} & \ldots & x_{D}^{1} \\
\vdots & \vdots & \vdots & \vdots & \vdots \\
x_{1}^{N p o p} & x_{2}^{N p o p} & x_{3}^{N p o p} & \ldots & x_{D}^{N p o p}
\end{array}\right]
$$

In the first step, Npop streams are created. Then, a number of best individuals Nsr (minimum values) are selected as the sea and rivers. The stream which has the minimum value among the others is considered as the sea. In fact, $N s r$ is the summation of the number of rivers (which is defined by the user) and a single sea. The rest of the population (Nstream) are considered as streams flowing into the rivers or may alternatively flow directly into the sea [14].

Depending on the magnitude of the flow, each river absorbs water from streams. Hence, the amount of water entering a river and/or the sea varies from stream to stream. In addition, rivers flow to the sea, which is the most downhill location. The designated streams for each river and the sea are calculated using the following [15]:

$$
\begin{aligned}
& N S_{n}=\operatorname{round}\left\{\left|\frac{\text { Cost }_{n}-\text { Cost }_{N s r+1}}{\sum_{n=1}^{N_{s r} C_{n}}}\right| \times N_{\text {Streams }}\right\}, \\
& n=1,2,3, \ldots, N_{s r}
\end{aligned}
$$

where $N S_{n}$ is the number of streams which flow into the specific rivers and the sea. For the exploitation phase of the WCA, new positions for streams and rivers have been suggested as follows [13]:

$$
\begin{aligned}
& X_{\text {stream }}(t+1)=X_{\text {stream }}(t)+\text { rand } \times C \times\left(X_{\text {sea }}(t)-X_{\text {stream }}(t)\right) \\
& X_{\text {stream }}(t+1)=X_{\text {stream }}(t)+\operatorname{rand} \times C \times\left(X_{\text {river }}(t)-X_{\text {stream }}(t)\right) \\
& X_{\text {river }}(t+1)=X_{\text {river }}(t)+\text { rand } \times C \times\left(X_{\text {sea }}(t)-X_{\text {river }}(t)\right)
\end{aligned}
$$

where $t$ is an iteration index, $1<C<2$, and the best value for $C$ may be chosen as 2 , and rand is a uniformly distributed random number between [0,1]. In (4) And (5) are for streams which flow into the sea and their corresponding rivers, respectively. If the solution given by a stream is more optimal than that of its connecting river, the positions of the river and stream are exchanged. A similar exchange can be performed for a river and the sea. The evaporation process operator is also introduced to avoid premature (immature) convergence to local optima (exploitation phase) [13]. Basically, evaporation causes sea water to evaporate as rivers/streams flow into the sea. This leads to new precipitation. Therefore, we have to check whether the river/stream is sufficiently close to the sea to enable the evaporation process to occur. The following criterion is utilized for the evaporation condition between a river and the sea [15]:

$$
\left\|X_{\text {sea }}^{t}-X_{\text {river }_{j}}^{t}\right\|<d_{\text {max }} \text { or rand }<0.1 j=1,2, \ldots, N_{s r}-1
$$


where $d_{\text {max }}$ is a small number close to zer o. After evaporation, the raining process is applied and new streams are formed in different locations. Indeed, the evaporation operator is responsible for the exploration phase in the WCA. Uniform random search is used to specify the new locations of the newly formed streams. A large value for $d_{\max }$ prevents additional searches and small values encourage the search intensity near the sea. Therefore, $d_{\max }$ controls the search intensity near the sea. The value of $d_{\max }$ adaptively decreases as follows [16]:

$$
d_{\text {max }}(t+1)=d_{\max }(t)-\frac{d_{\max }(t)}{\text { max.iteration }} \quad t=1,2, \ldots, \text { max. iteration }
$$

For more details about the metaheuristic approach, we can see $[17,18]$.

\section{THE PROPOSED ALGORITHM FOR OPTIMIZATION PROBLEM}

In this section, we propose a new hybrid algorithm WCA-PSO is collaborative combinations of the WCA and PSO techniques. In this hybrid, firstly, WCA explores the search place in order to either isolate the most promising region of the search space. Secondly, to improve global search and avoid trapping into local optima, it is introduced PSO to explore search space (starting with the solution obtained by WCA) and find new population, which is closer to optimal solution. Further, WCA will be obtained the best model parameters vector. The structure of the hybrid WCA-PSO is shown by the following Algorithm 1.

\section{Algorithm 1: Hybrid WCA-PSO Algorithm}

Input: Objective function min or $\max \boldsymbol{f}(\boldsymbol{x})$

Output: The optimal solutions for each variable and the optimal cost

Determine the initial parameters of WCA $\mathbf{N p o p}, \mathbf{N s}$, ,: and Maximum Iteration.

Generate randomly initial population and Forming the initial sea, rivers and streams.

Calculate the fitness of each initial population by using $\boldsymbol{f}(\boldsymbol{x})$

Computing the corresponding flow intensity of river and sea

While $(t<$ Maximum Iteration)

For i=1: Population size $\left(\boldsymbol{N}_{\text {pop }}\right)$

Stream flows to its corresponding rivers and sea

Calculate the objective function of the generated stream

If $\mathrm{F} \_$New_Stream $<$F_river

River $=$ New_stream

If F_New_stream < F_Sea

End

Sea $=$ New_Stream

End

River flows to the sea

Calculate the objective function of the generated river

If F_New_River < F_Sea

End

Sea $=$ New River

End

For i=1: number of rivers $\left(\boldsymbol{N}_{\boldsymbol{s r}}\right)$

If (distance (Sea and River) $<\boldsymbol{d}_{\max }$ ) or $($ rand $<0.1$ )

New streams are created

End

End

End while

Reduce the $\boldsymbol{d}_{\max }$

Store the best solution of water cycle as the initial locations $\boldsymbol{x}_{\boldsymbol{i}}$ of n particles

Initialize velocity $\boldsymbol{v} \boldsymbol{i}$ of $n$ particles.

Find $\boldsymbol{g}^{*}$ from objective function $\boldsymbol{f}(\boldsymbol{x})$ (at $t=0$ )

while (criterion)

for loop over all $n$ particles and all $d$ dimensions

Generate new velocity $\boldsymbol{v}_{i d}^{t+1}$, Calculate new locations $X_{i d}^{t+1}=X_{i d}^{t}+V_{i d}^{t+1}$

Evaluate objective functions at new locations $\boldsymbol{X}_{\boldsymbol{i d}}^{\boldsymbol{t + 1}}$

Find the current best for each particle

End for 
Find the current global best $\boldsymbol{g}^{*}$

Update $\boldsymbol{t}=\boldsymbol{t}+\mathbf{1}$

End while

Display result.

\section{EVALUATION OF THE PROPOSED ALGORITHM WCA-PSO}

The main objective of this section is the evaluation of the proposed algorithm WCA-PSO by benchmark problems. We evaluate the performance of the proposed algorithm WCA-PSO by the numerical simulation based on some Benchmark problems [17, 18] to investigate the performances of the proposed algorithms. The functions name with global optimum, search ranges and initialization ranges of the test functions are presented in Table 1. In these problems, the essential parameters of WCA are number of rivers and sea $N s r=4$. And the PSO constants are $C_{1}=C_{2}=2$, the population size for all algorithms is 50 that are the same used for WCA-PSO algorithm. The results of all algorithms are conducted from 20 independent run for each problem. All the experiments were performed on a Windows 10 Ultimate 64-bit operating system; processor Intel Core i7 760 running at $2.40 \mathrm{GHz} ; 8 \mathrm{~GB}$ of RAM and code was implemented in MATLAB 2016.

From Table 2 and Figure 1, the results show that the proposed hybrid algorithm WCA-PSO overcome the traditional PSO and traditional WCA solutions. The results explain that WCA-PSO is robust and competitive with the state-of the-art well-known evolutionary algorithms. We note that the performance of WCA-PSO is significantly superior to all the present algorithms for all functions according to the experimental results. The mean and the difference between the best value and worst value of the result obtained by WCA-PSO were small compared to the results we have obtained from other algorithms in functions F03, F04, F06, F07, F08 and F10. General, the performance of WCA-PSO is highly competitive with other algorithms.

Table 1. The benchmark functions

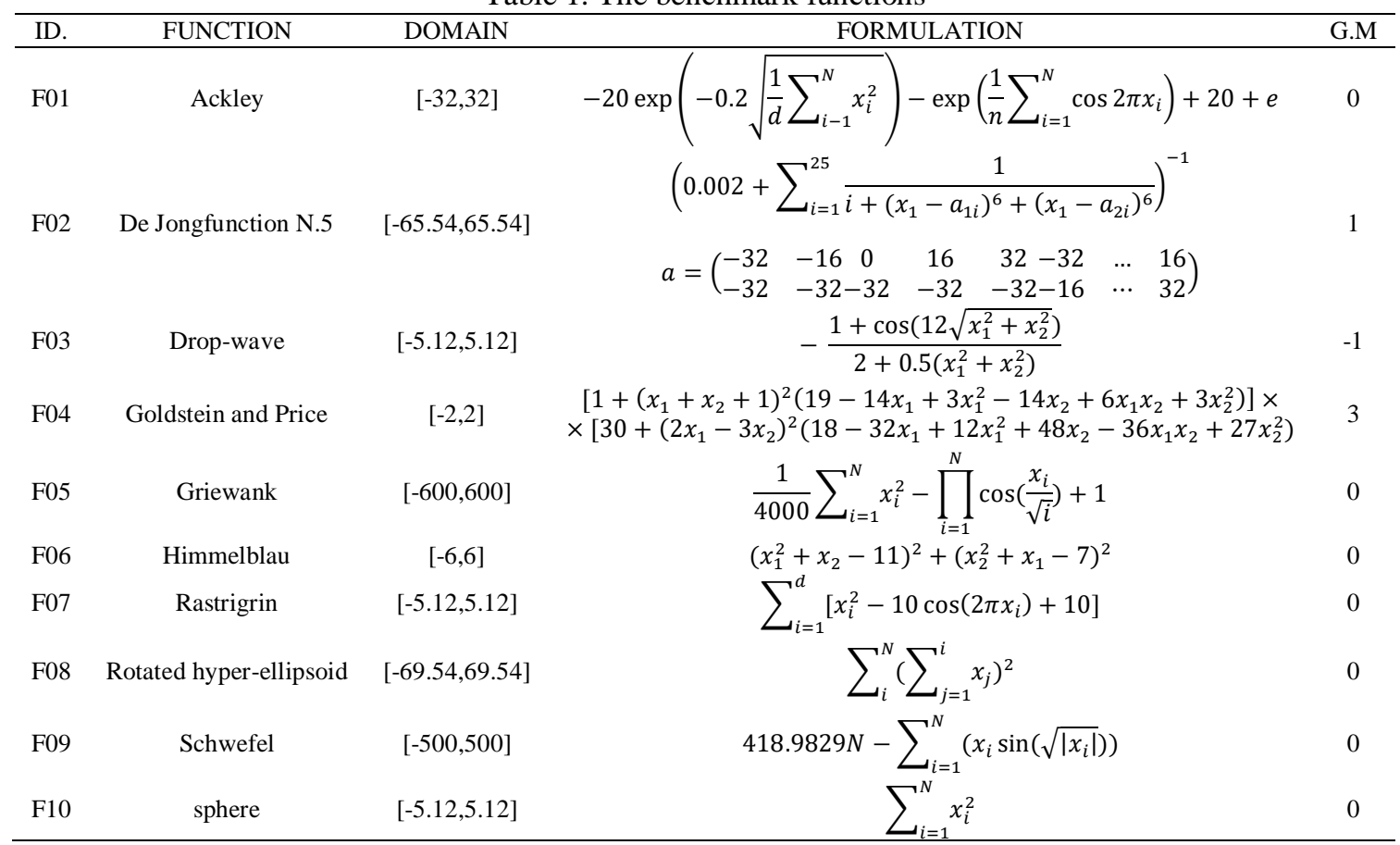

Table 2. The optimal solution results of proposed algorithm and other algorithms

\begin{tabular}{cccccc}
\hline ID. & Algorithm & Min & Max & Mean & Stander Deviation \\
\hline \multirow{3}{*}{ F01 } & PSO & $8.19 \mathrm{E}-16$ & $8.88 \mathrm{E}-16$ & $8.26 \mathrm{E}-16$ & $2.1327 \mathrm{E}-17$ \\
& WCA & $1.17 \mathrm{E}-16$ & $8.89 \mathrm{E}-16$ & $8.19 \mathrm{E}-16$ & $3.10 \mathrm{E}-16$ \\
& WCA-PSO & $8.88 \mathrm{E}-16$ & $8.88 \mathrm{E}-16$ & $8.88 \mathrm{E}-16$ & $1.01169 \mathrm{E}-31$ \\
\multirow{2}{*}{ F02 } & PSO & $9.88 \mathrm{E}-01$ & $2.98 \mathrm{E}+00$ & $1.74 \mathrm{E}+00$ & 0.844551579 \\
& WCA & $9.98 \mathrm{E}-01$ & $1.98 \mathrm{E}+00$ & $9.98 \mathrm{E}-01$ & 0.302118 \\
& WCA-PSO & $9.98 \mathrm{E}-01$ & $1.99 \mathrm{E}+00$ & $9.98 \mathrm{E}-01$ & 0.499609 \\
\hline
\end{tabular}




\begin{tabular}{cccccc}
\hline \multirow{2}{*}{ F03 } & PSO & $-1.00 \mathrm{E}+00$ & $-9.98 \mathrm{E}-01$ & $-1.00 \mathrm{E}+00$ & 0.000732695 \\
& WCA & $-1.00 \mathrm{E}+00$ & $-9.99 \mathrm{E}-01$ & $-1.00 \mathrm{E}+00$ & 0.00030779 \\
& WCA-PSO & $-1.00 \mathrm{E}+00$ & $-1.00 \mathrm{E}+00$ & $-1.00 \mathrm{E}+00$ & $0.00 \mathrm{E}+00$ \\
F04 & PSO & $3.00 \mathrm{E}+00$ & $3.01 \mathrm{E}+00$ & $3.00 \mathrm{E}+00$ & 0.004396171 \\
& WCA & $3.00 \mathrm{E}+00$ & $3.00 \mathrm{E}+00$ & $3.00 \mathrm{E}+00$ & 0.000158 \\
& WCA-PSO & $3.00 \mathrm{E}+00$ & $3.00 \mathrm{E}+00$ & $3.00 \mathrm{E}+00$ & $0.00 \mathrm{E} 00$ \\
F05 & PSO & $0.00 \mathrm{E} 00$ & $7.40 \mathrm{E}-03$ & $1.85 \mathrm{E}-03$ & 0.003285759 \\
& WCA & $-7.39 \mathrm{E}-03$ & $4.00 \mathrm{E}-01$ & $4.74 \mathrm{E}-03$ & 0.01976451 \\
& WCA-PSO & $0.00 \mathrm{E}+00$ & $0.00 \mathrm{E}+00$ & $0.00 \mathrm{E}+00$ & $0.00 \mathrm{E}+00$ \\
F06 & PSO & $0.00 \mathrm{E}+00$ & $7.89 \mathrm{E}-31$ & $1.58 \mathrm{E}-31$ & $3.23635 \mathrm{E}-31$ \\
& WCA & $0.00 \mathrm{E}+00$ & $7.89 \mathrm{E}-31$ & $0.00 \mathrm{E}+00$ & $1.88793 \mathrm{E}-31$ \\
& WCA-PSO & $0.00 \mathrm{E}+00$ & $7.89 \mathrm{E}-31$ & $0.00 \mathrm{E}+00$ & $3.5 \mathrm{E}-31$ \\
F07 & PSO & $0.00 \mathrm{E}+00$ & $1.78 \mathrm{E}-14$ & $2.66 \mathrm{E}-15$ & $6.50633 \mathrm{E}-15$ \\
& WCA & $0.00 \mathrm{E}+00$ & $3.55 \mathrm{E}-15$ & $1.78 \mathrm{E}-15$ & $1.04 \mathrm{E}-15$ \\
& WCA-PSO & $0.00 \mathrm{E}+00$ & $0.00 \mathrm{E}+00$ & $0.00 \mathrm{E}+00$ & $0.00 \mathrm{E}+00$ \\
F08 & PSO & $1.64 \mathrm{E}-220$ & $9.50 \mathrm{E}-114$ & $4.75 \mathrm{E}-115$ & $2.1242 \mathrm{E}-114$ \\
& WCA & $3.97 \mathrm{E}-223$ & $4.37 \mathrm{E}-118$ & $3.35 \mathrm{E}-218$ & $9.65 \mathrm{E}-119$ \\
& WCA-PSO & $6.43 \mathrm{E}-225$ & $8.74 \mathrm{E}-206$ & $4.94 \mathrm{E}-217$ & $0.00 \mathrm{E} 00$ \\
F09 & PSO & $2.55 \mathrm{E}-05$ & $1.18 \mathrm{E}+02$ & $4.74 \mathrm{E}+01$ & 59.53005127 \\
& WCA & $2.55 \mathrm{E}-05$ & $1.18 \mathrm{E}+02$ & $2.55 \mathrm{E}-05$ & 43.38946163 \\
& WCA-PSO & $2.55 \mathrm{E}-05$ & $1.18 \mathrm{E}+02$ & $2.55 \mathrm{E}-05$ & 60.44877 \\
& PSO & $1.05 \mathrm{E}-228$ & $4.08 \mathrm{E}-112$ & $2.07 \mathrm{E}-113$ & $9.1 \mathrm{E}-113$ \\
& WCA & $1.29 \mathrm{E}-224$ & $3.78 \mathrm{E}-117$ & $2.59 \mathrm{E}-220$ & $8.4051 \mathrm{E}-118$ \\
& WCA-PSO & $4.31 \mathrm{E}-227$ & $7.78 \mathrm{E}-209$ & $3.58 \mathrm{E}-220$ & $0.00 \mathrm{E}+00$ \\
\hline
\end{tabular}
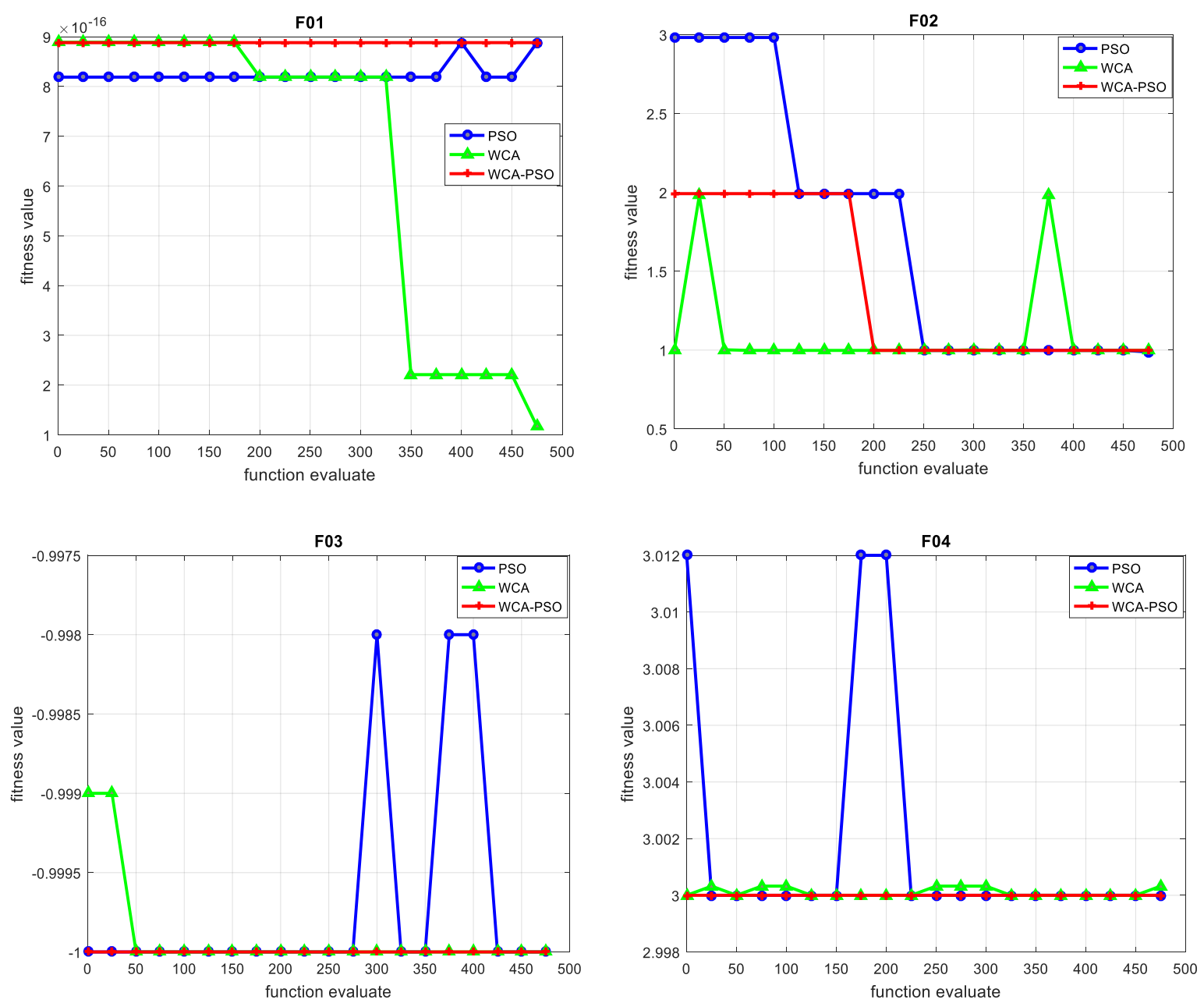

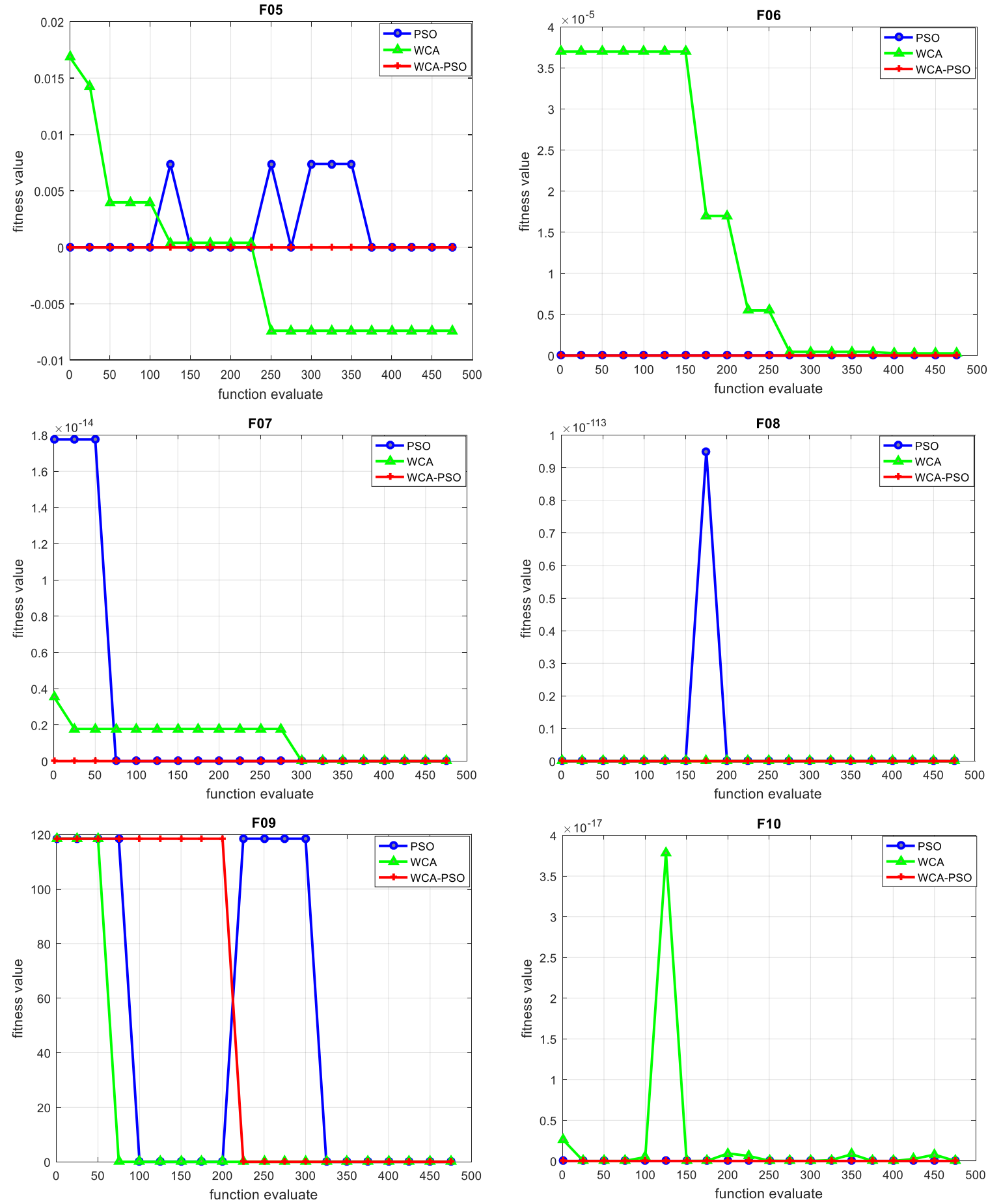

Figure 1. F01:F10 the convergence rate of the function error values on 10 function (continue)

\section{MULTIOBJECTIVE FUZZY OPTIMIZATION MODEL FOR AQUIFER MANAGEMENT}

O. M. Saad et al. [19] formulated the fuzzy multiobjective optimization model for the aquifer management, in three dimensions as follows:

(FMOM):

$$
\begin{aligned}
& \max \sum_{i=1}^{n} \sum_{j=1}^{m} \sum_{k=1}^{l} \tilde{L}_{i j k} \\
& \max \sum_{i=1}^{n} \sum_{j=1}^{m} \sum_{k=1}^{l} \widetilde{W}_{i j k}
\end{aligned}
$$




$$
\min \sum_{i=1}^{n} \sum_{j=1}^{m} \sum_{k=1}^{l}\left[\theta\left(\tilde{L}_{i j k}\right)^{\delta}+\gamma+\beta\right]
$$

Subject to:

$$
\begin{aligned}
& \sum_{i=1}^{n} \sum_{j=1}^{m} \sum_{k=1}^{l} \widetilde{W}_{i j k} \geq \text { Demand } \\
& A_{n \times n}(\widetilde{T}) \widetilde{L}_{n \times 1} \leq \widetilde{b}_{n \times 1}+\widetilde{W}_{n \times 1} \\
& W_{l} \leq W^{\alpha} \leq W_{u} \\
& \mathrm{~T}, \mathrm{~L}, \mathrm{~b}, \mathrm{~W} \geq 0
\end{aligned}
$$

where $A_{n \times n}\left(\widetilde{T)}\right.$ is the matrix of fuzzy head coefficients which is a function of the transmissivity, $\tilde{L}_{n \times 1}$ is a fuzzy vector of unknown head values at each node, $\tilde{b}_{n \times 1}$ is a fuzzy vector containing the boundary head conditions, $\widetilde{W}_{n \times 1}$ is a fuzzy vector which associated with the pumping rate, $\theta=5543, \delta=0.299, \gamma$ is the per-well drilling cost (\$/well), and $\beta$ is the pump cost (\$/pump), represents the presence of fuzzy numbers within the matrices or vectors. Thus, model output will be expressed by membership functions that describe the head values as fuzzy variables.

Definition 1

The $\propto$-cut (alpha cut) is a method to generate a crisp interval corresponding to a given membership value. The crisp set contains all elements of the universal set are greater than or equal to the specified value. A $\propto$-cut set of triangular fuzzy number $\tilde{a}=\left(a^{l}, a^{m}, a^{r}\right)$ is defined as [20].

$$
\tilde{a}(x)=\left\{x: \mu_{\tilde{a}}(x) \geq \alpha\right\}
$$

Thus, for any $\alpha \in[0,1]$, we can obtain a $\propto$-cut set of triangular fuzzy number $\tilde{a}$, which is an interval, denoted by:

$$
\begin{aligned}
& \tilde{a}(\alpha)=\left[a^{l}(\alpha), a^{R}(\alpha)\right] ; \\
& a^{l}(\alpha)=\alpha a^{m}+(1-\alpha) a^{l} \\
& a^{R}(\alpha)=\alpha a^{m}+(1-\alpha) a^{R}
\end{aligned}
$$

where $a^{l}(\alpha)$ is a left number, $a^{R}(\alpha)$ is a right number and $a^{m}$ is a mean of $a^{l}$ and $a^{R}$, as shown at Figure 2.

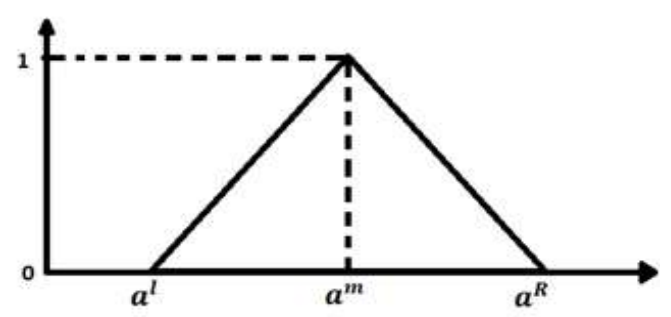

Figure 2. Triangle Membership function of fuzzy number

For certain values $\alpha_{T}^{*}, \alpha_{h}^{*}, \alpha_{b}^{*}, \alpha_{q}^{*}$ to be in the interval [0,1], the problem (FMOM) (9a) and (10c) can be reformulated as the following fuzzy multiobjective fuzzy optimization model for the aquifer management, in three-dimensions as follows:

$(\alpha-F M O M)$ :

$$
\begin{aligned}
& \max \sum_{i=1}^{n} \sum_{j=1}^{m} \sum_{k=1}^{l} L_{i j k} \\
& \max \sum_{i=1}^{n} \sum_{j=1}^{m} \sum_{k=1}^{l} W_{i j k} \\
& \min \sum_{i=1}^{n} \sum_{j=1}^{m} \sum_{k=1}^{l}\left[\theta\left(L_{i j k}\right)^{\delta}+\gamma+\beta\right]
\end{aligned}
$$


Subject to:

$$
\begin{aligned}
& \sum_{i=1}^{n} \sum_{j=1}^{m} \sum_{k=1}^{l} W_{i j k} \geq \text { Demand } \\
& A_{n \times n}(T) L_{n \times 1} \leq b_{n \times 1}+W_{n \times 1} \\
& T_{l} \leq T^{\alpha} \leq T_{u} \\
& L_{l} \leq L^{\alpha} \leq \boldsymbol{L}_{u} \\
& \boldsymbol{b}_{l} \leq \boldsymbol{b}^{\alpha} \leq \boldsymbol{b}_{u} \\
& \boldsymbol{W}_{l} \leq \boldsymbol{W}^{\alpha} \leq \boldsymbol{W}_{\boldsymbol{u}} \\
& \mathrm{T}, \mathrm{L}, \mathrm{b}, \mathrm{W} \geq \mathbf{0}
\end{aligned}
$$

where $T_{l}, T_{u}, L_{l}, L_{u}, b_{l}, b_{u}, W_{l}$ and $W_{u}$ are lower and upper bounds on $\mathrm{T}, \mathrm{L}, \mathrm{b}$ and $\mathrm{W}$, respectively.

In (13b) can be calculated from the partial differential equation describing the system of interest in three dimensions as follows:

$$
\frac{\partial}{\partial x}\left(T \frac{\partial L}{\partial x}\right)+\frac{\partial}{\partial y}\left(T \frac{\partial L}{\partial y}\right)+\frac{\partial}{\partial z}\left(T \frac{\partial L}{\partial z}\right)=W
$$

this can be decoded on the following equation:

$$
L_{i+1, j, k}+L_{i-1, j, k}+L_{i, j+1, k}+L_{i, j-1, k}+L_{i, j, k+1}+L_{i, j, k-1}-6 L_{i, j, k}=\frac{(\Delta x)^{2}}{T} W_{i, j, k}
$$

the lower bound $L_{l}$ and the upper bound $L_{u}$ can be calculate using the following nonlinear programming problems [19].

$L_{l}^{*}$ :

Subject to

$$
\text { mi n } L_{i, j, k}^{\alpha}
$$

$$
\begin{aligned}
& A\left(T^{\alpha}\right)(L)^{\alpha}=b^{\alpha} \\
& T_{i, j, k}^{\alpha} \leq T^{\alpha} \leq \overline{T_{i, j, k}^{\alpha}} \\
& \underline{b_{i, j, k}^{\alpha}} \leq b^{\alpha} \leq \overline{b_{i, j, k}^{\alpha}}
\end{aligned}
$$

$L_{u}^{*}:$

Subject to

$$
\max L_{i, j, k}^{\alpha}
$$

$$
\begin{aligned}
& A\left(T^{\alpha}\right)(L)^{\alpha}=b^{\alpha} \\
& \underline{T_{i, j, k}^{\alpha}} \leq T^{\alpha} \leq \overline{T_{i, j, k}^{\alpha}} \\
& \underline{b_{i, j, k}^{\alpha}} \leq b^{\alpha} \leq \overline{b_{i, j, k}^{\alpha}}
\end{aligned}
$$

The lower bound $W_{l}$ and the upper bound $W_{u}$ can be calculated using the following nonlinear programming problems:

$W_{l}^{*}:$

Subject to

$$
\min W_{i, j, k}^{\alpha}
$$

$$
\begin{aligned}
& A\left(T^{\alpha}\right)(L)^{\alpha}=b^{\alpha} \\
& \underline{T_{i, j, k}^{\alpha} \leq T^{\alpha} \leq \overline{T_{i, j, k}^{\alpha}}}
\end{aligned}
$$




$$
\underline{b_{i, j, k}^{\alpha}} \leq b^{\alpha} \leq \overline{b_{i, j, k}^{\alpha}}
$$

$W_{u}^{*}:$

Subject to

$$
\max W_{i, j, k}^{\alpha}
$$

$$
\begin{aligned}
& A\left(T^{\alpha}\right)(L)^{\alpha}=b^{\alpha} \\
& \frac{T_{i, j, k}^{\alpha}}{T_{i, j}^{\alpha}} \leq T^{\alpha} \leq \overline{T_{i, j, k}^{\alpha}} \\
& b_{i, j, k}^{\alpha} \leq \overline{b_{i, j, k}^{\alpha}}
\end{aligned}
$$

where $\underline{T_{i, j, k}^{\alpha}}, \overline{T_{i, j, k}^{\alpha}}, \underline{b_{i, j, k}^{\alpha}}, \overline{b_{i, j, k}^{\alpha}}$, are the lower and upper bounds on $T_{i, j, k}^{\alpha}, b_{i, j, k}^{\alpha}$ respectively , $T^{\alpha}$ is the vector of transmissivities at the specified $\alpha$-cut level, $A\left(T^{\alpha}\right)$ is the matrix of head coefficients which is a function of $T^{\alpha}, b^{\alpha}$ is the right hand side vector containing the boundary conditions and source/sink terms and $L^{\alpha}$ is the vector of unknown heads at the specified $\alpha$ - level cut. Thus, to calculate fuzzy head at a specific node two nonlinear programming problems are considered "the lower and upper bound of the unknown head can be calculated by optimization the two models mathematical, and then we find the optimal solutions using any suitable software, is obtained. For more details about the multiobjective linear and non-linear programming approach, we can see [21-26].

\section{RESULTS AND DISCUSSIONS}

Supposing the leakage of flux into or out of aquifer and the well diameters are to be negligible, well losses are negligible, and the head in the well is measured from the surface of the producing layer which is considered as a horizontal datum. Input data for the simulation model includes fuzzy transmissivity values at each node, fuzzy number head boundary conditions, transmissivity of boundary nodes, the discharge rate of the well and the basic simulation parameters. The heads on boundaries are fuzzy number values of 50 to 60 $\mathrm{m}$. The demand is $500 \mathrm{~m} /$ day and the upper bound of the total water production is 2000 .

$$
\begin{aligned}
& \Delta x=\Delta y=\Delta z=10 m,, T \in[200,300] \mathrm{m} 2 / \text { day, } \\
& \gamma=13.511(\$ / \text { well }), \beta=3832(\$ / \text { pump }), \alpha \in[0,1]
\end{aligned}
$$

Case 1

Triangle membership function ( $n=m=l=2)$,

We compute the lower and upper bound of head and pump rate for each node using the (16-19), and Set $\alpha=0.4$, using the triangle membership function and (11) to get the lower and upper bound of head and pump rate for each node, and $220 \leq \mathrm{T} \leq 280,52 \leq \mathrm{b} \leq 58$, Table 3 and Table 4 show the solution of the model.

Table 3. Results of water head and pumping rate for each wall

\begin{tabular}{cccccccc}
\hline $\begin{array}{c}\text { Water } \\
\text { head }(\mathrm{L})\end{array}$ & $\begin{array}{c}\text { Rang of } \\
\text { water head }\end{array}$ & $\begin{array}{c}\text { Rang of water } \\
\text { head at } \alpha=0.4\end{array}$ & $\begin{array}{c}\text { Optimal } \\
\text { water head }\end{array}$ & $\begin{array}{c}\text { Pump } \\
\text { rate }(\mathrm{W})\end{array}$ & $\begin{array}{c}\text { Rang of } \\
\text { pumping rate }\end{array}$ & $\begin{array}{c}\text { Rang of pumping } \\
\text { rate at } \alpha=0.4\end{array}$ & $\begin{array}{c}\text { Optimal } \\
\text { pump rate }\end{array}$ \\
\hline $\boldsymbol{L}_{\mathbf{1 1 1}}$ & {$[0,50.49]$} & {$[10.10,40.40]$} & 40.40 & $W_{111}$ & 0 & 0 & 0 \\
$\boldsymbol{L}_{\mathbf{1 1 2}}$ & {$[0,50.48]$} & {$[10.10,40.39]$} & 40.39 & $W_{112}$ & {$[0,528.76]$} & {$[105.7,423.24]$} & 423.24 \\
$\boldsymbol{L}_{\mathbf{1 2 1}}$ & {$[0,50.48]$} & {$[10.10,40.39]$} & 40.24 & $W_{121}$ & {$[0,221.25]$} & {$[44.25,177]$} & 177 \\
$\boldsymbol{L}_{\mathbf{1 2 2}}$ & {$[0,50.31]$} & {$[10.06,40.2]$} & 40.39 & $W_{122}$ & 0 & 0 & 0 \\
$\boldsymbol{L}_{\mathbf{2 1 1}}$ & {$[0,51.40]$} & {$[10.28,41.12]$} & 41.12 & $W_{211}$ & {$[0,400.44]$} & {$[420.35,480]$} & 480 \\
$\boldsymbol{L}_{\mathbf{2 1 2}}$ & {$[0,50.76]$} & {$[10.15,40.61]$} & 40.61 & $W_{212}$ & 0 & 0 & 0 \\
$\boldsymbol{L}_{\mathbf{2 2 1}}$ & {$[0,50.76]$} & {$[10.15,40.61]$} & 40.61 & $W_{221}$ & {$[0,384.95]$} & {$[76.99,307.96]$} & 307.96 \\
$\boldsymbol{L}_{\mathbf{2 2 2}}$ & {$[0,51.06]$} & {$[10.21,40.85]$} & 40.85 & $W_{222}$ & {$[0,464.59]$} & {$[92.9,371.68]$} & 371.68 \\
\hline
\end{tabular}


Table 4. Comparison among PSO, WCA and WCA-PSO on multiobjective optimization model for aquifer management

\begin{tabular}{lccc}
\hline & PSO & WCA & WCA-PSO \\
\hline Max sum of water head & 324.61 & 324.61 & 324.61 \\
Max total water production & 1760 & 1760 & 1760 \\
Minimum cost & 165265.88 & 165265.88 & 165265.88 \\
Number of iterations & 500 & 100 & 50 \\
CPU time (sec) & 2.51 & 0.21 & 0.35 \\
\hline
\end{tabular}

Case 2

Triangle membership function $(n=m=l=3$ )

We compute the lower and upper bound of head and pump rate for each node using the (16-19), and Set $\alpha=0.4$, using the triangle membership function and (11) to get $220 \leq \mathrm{T} \leq 280$ and $52 \leq \mathrm{b} \leq 58$. Table 5 and Table 6 show the solution of the model. Tables 3-6 show that the WCA-PSO hybrid algorithm overcomes the other optimization algorithms according to number of iterations and the CPU time for the two cases. It is also proving that increasing the CPU time when the size of the issue is greater and it will be occurs when choosing $n, m$ and $l$ larger.

Table 5. Results of water head and pumping rate for each wall

\begin{tabular}{|c|c|c|c|c|c|c|c|}
\hline $\begin{array}{c}\text { Water } \\
\text { head }(\mathrm{L})\end{array}$ & $\begin{array}{c}\text { Rang of } \\
\text { water head }\end{array}$ & $\begin{array}{l}\text { Rang of water } \\
\text { head at } \alpha=0.4\end{array}$ & $\begin{array}{c}\text { Optimal } \\
\text { water head }\end{array}$ & $\begin{array}{l}\text { Pump } \\
\text { rate }(\mathrm{W})\end{array}$ & $\begin{array}{c}\text { Rang of } \\
\text { pumping rate }\end{array}$ & $\begin{array}{l}\text { Rang of pumping } \\
\text { rate at } \alpha=0.4\end{array}$ & $\begin{array}{l}\text { Optimal } \\
\text { pumprate }\end{array}$ \\
\hline$L_{111}$ & {$[0,57.80]$} & {$[11.57,46.28]$} & 46.28 & $W_{111}$ & {$[0,487.20]$} & {$[97.44,389.7]$} & 389.76 \\
\hline$L_{112}$ & {$[0,57.70]$} & {$[11.54,46.16]$} & 46.16 & $W_{112}$ & {$[0,324.80]$} & {$[64.96,259.8]$} & 259.84 \\
\hline$L_{113}$ & {$[0,57.66]$} & {$[11.53,46.13]$} & 46.13 & $W_{113}$ & {$[0,487.20]$} & {$[97.44,389.7]$} & 389.76 \\
\hline$L_{121}$ & {$[0,57.69]$} & {$[11.54,46.15]$} & 46.15 & $W_{121}$ & {$[0,324.80]$} & {$[64.96,259.8]$} & 259.84 \\
\hline$L_{122}$ & {$[0,57.33]$} & {$[11.47,45.86]$} & 45.86 & $W_{122}$ & {$[0,162.40]$} & {$[32.48,129.9]$} & 129.92 \\
\hline$L_{123}$ & {$[0,57.15]$} & {$[11.43,45.72]$} & 45.72 & $W_{123}$ & {$[0,324.80]$} & {$[64.96,259.8]$} & 259.84 \\
\hline$L_{131}$ & {$[0,57.66]$} & {$[11.53,46.13]$} & 46.13 & $W_{131}$ & {$[0,487.20]$} & {$[97.44,389.7]$} & 389.76 \\
\hline$L_{132}$ & {$[0,57.15]$} & {$[11.43,45.72]$} & 45.72 & $W_{132}$ & {$[0,324.80]$} & {$[64.96,259.8]$} & 259.84 \\
\hline$L_{133}$ & {$[0,56.63]$} & {$[11.33,45.30]$} & 45.30 & $W_{133}$ & {$[0,487.20]$} & {$[97.44,389.7]$} & 389.76 \\
\hline$L_{211}$ & {$[0,57.70]$} & {$[11.54,46.16]$} & 46.16 & $W_{211}$ & {$[0,324.80]$} & {$[64.96,259.8]$} & 259.84 \\
\hline$L_{212}$ & {$[0,57.3]$} & {$[11.47,45.86]$} & 45.86 & $W_{212}$ & {$[0,162.40]$} & {$[32.48,129.9]$} & 129.92 \\
\hline$L_{213}$ & {$[0,57.15]$} & {$[11.43,45.72]$} & 45.72 & $W_{213}$ & {$[0,324.80]$} & {$[64.96,259.8]$} & 259.84 \\
\hline$L_{221}$ & {$[0,57.33]$} & {$[11.47,45.86]$} & 45.86 & $W_{221}$ & {$[0,162.40]$} & {$[32.48,129.9]$} & 129.92 \\
\hline$L_{222}$ & {$[0,56.29]$} & {$[11.26,45.03]$} & 45.03 & $W_{222}$ & 0 & 0 & 0 \\
\hline$L_{223}$ & {$[0,55.26]$} & {$[11.05,44.21]$} & 44.21 & $W_{223}$ & {$[0,162.40]$} & {$[32.48,129.9]$} & 129.92 \\
\hline$L_{231}$ & {$[0,57.15]$} & {$[11.43,45.72]$} & 45.72 & $W_{231}$ & {$[0,324.80]$} & {$[64.96,259.8]$} & 259.84 \\
\hline$L_{232}$ & {$[0,55.26]$} & {$[11.05,44.21]$} & 44.21 & $W_{232}$ & {$[0,162.40]$} & {$[32.48,129.9]$} & 129.92 \\
\hline$L_{233}$ & {$[0,51.48]$} & {$[10.30,41.18]$} & 41.18 & $W_{233}$ & {$[0,324.80]$} & {$[64.96,259.8]$} & 259.84 \\
\hline$L_{311}$ & {$[0,57.66]$} & {$[11.53,46.13]$} & 46.13 & $W_{311}$ & {$[0,487.20]$} & {$[97.44,389.7]$} & 389.76 \\
\hline$L_{312}$ & {$[0,57.15]$} & {$[11.43,45.72]$} & 45.72 & $W_{312}$ & {$[0,324.80]$} & {$[64.96,259.8]$} & 259.84 \\
\hline$L_{313}$ & {$[0,56.6]$} & {$[11.33,45.30]$} & 45.30 & $W_{313}$ & {$[0,487.20]$} & {$[97.44,389.7]$} & 389.76 \\
\hline$L_{321}$ & {$[0,57.15]$} & {$[11.43,45.72]$} & 45.72 & $W_{321}$ & {$[0,324.80]$} & {$[64.96,259.8]$} & 259.84 \\
\hline$L_{322}$ & {$[0,55.26]$} & {$[11.05,44,21]$} & 44. 21 & $W_{322}$ & {$[0,162.40]$} & {$[32.48,129.9]$} & 129.92 \\
\hline$L_{323}$ & {$[0,51.48]$} & {$[10.30,41.18]$} & 41.18 & $W_{323}$ & {$[0,324.80]$} & {$[64.96,259.8]$} & 259.84 \\
\hline$L_{331}$ & {$[0,56.63]$} & {$[11.33,45.30]$} & 45.30 & $W_{331}$ & {$[0,487.20]$} & {$[97.44,389.7]$} & 389.76 \\
\hline$L_{332}$ & {$[0,51.48]$} & {$[10.30,41.18]$} & 41.18 & $W_{332}$ & {$[0,324.80]$} & {$[64.96,259.8]$} & 259.84 \\
\hline$L_{333}$ & {$[0,58.00]$} & {$[11.60,46.40]$} & 46.40 & $W_{333}$ & {$[0,486.20]$} & {$[97.44,389.7]$} & 389.76 \\
\hline
\end{tabular}

Table 6. Comparison among PSO, WCA and WCA-PSO on multiobjective optimization model for aquifer management

\begin{tabular}{lccc}
\hline & PSO & WCA & WCA-PSO \\
\hline Max sum of water heads $\left(\mathrm{m}^{3}\right)$ & 1218.57 & 1218.57 & 1218.57 \\
Max sum of water pumping & 7015.68 & 7015.68 & 7015.68 \\
Minimum cost (\$) & 571291.1 & 571291.1 & 571291.1 \\
Number of iterations & 20000 & 500 & 100 \\
CPU time (sec.) & 50.32 & 1.20 & 0.69 \\
\hline
\end{tabular}

\section{CONCLUSION}

In this work, we introduced a novel hybrid algorithm using water cycle algorithm (WCA) and particle swarm optimization (PSO). The performance of the novel hybrid algorithm WCA-PSO we evaluated to solve 10 benchmark problems chosen from literature. The simulation results and comparison with pure WCA and PSO algorithms confirmed the effectiveness of the proposed algorithm WCA-PSO for solving 
various benchmark optimization functions. Finally, we solved the problem of managing underground water aquifers by WCA, PSO and the hybrid optimization WCA-PSO. The experimental results analysis and statistical tests proved that the hybrid algorithm WCA-PSO overcomes the other algorithms. In future work, we can improve this work by using the different metaheuristic algorithms with other mathematical models.

\section{REFERENCES}

[1] Y. Xin-She, "Engineering Optimization: An Introduction with Metaheuristic Application," Wiley, 2010.

[2] H. A. Abbass and R. Sarker, "The Pareto diffential evolution algorithm," Int. J. Artificial Intelligence Tools, vol. 11, no. 4, pp. 531-552, 2002.

[3] Y. Xin-She, "Nature-inspired Metaheuristic Algorithms," Luniver Press, 2008.

[4] K. Deb, "Multi-Objective optimization using evolutionary algorithms," New York: John Wiley \& Sons, 2001.

[5] Y. Xin-She, K. Mehmet and H. Xingshi, "Multi-objective Flower Algorithm for Optimization," International Conference on Computational Science, ICCS 2013, 2013.

[6] Kennedy J., Eberhart R., "Particle swarm optimization," Proc IEEE Int Conf Neural Netw 4, pp. 1942-1948, 1995.

[7] Shi Y., Eberhart R., "A modified particle swarm optimizer," In: Proceedings of IEEE World Congress on computational intelligence. The 1998 I.E. international conference on evolutionary computation, pp 69-73, 1998.

[8] David S., "The water cycle, illustrations by John Yates," New York: Thomson Learning, 1993.

[9] Eskandar H., Sadollah A., Bahreininejad A., \& Hamdi M., "Water cycle algorithm-A novel metaheuristic optimization method for solving constrained engineering optimization problems. Computers \& Structures, vol. 110-111, pp. 151-166, 2012.

[10] Engelbrecht A.P., "Computational Intelligence: An Introduction,” John Wiley \& Sons, England, 2002.

[11] Kennedy J., And Eberhart R., "Particle swarm optimization," Proceedings of IEEE International Conference on Neural Networks, Piscataway, NJ.pp. 1942-1948, 1995.

[12] Shi Y., "Particle Swarm Optimization," In Proceedings of the International Machine Learning and Cybernetics, vol. 4, pp. 2236-2241, 2004.

[13] Eskandar H., Sadollah A., Bahreininejad A., \& Hamdi M., "Water cycle algorithm-A novel metaheuristic optimization method for solving constrained engineering optimization problems," Computers \& Structures, vol. 110-111, pp. 151-166, 2012.

[14] Sadollah A., Eskandar H., Bahreininejad A., \& Kim J. H., "Water cycle, mine blast and improved mine blast algorithms for discrete sizing optimization of truss structures," Computers \& Structures, vol. 149, pp. 1-16, 2015.

[15] Sadollah A., Eskandar H., Bahreininejad A., \& Kim J. H., "Water cycle algorithm with evaporation rate for solving constrained and unconstrained optimization problems," Applied Soft Computing, vol. 30, pp. 58-71, 2015.

[16] Heidari A. A., Abbaspour R. A., \& Jordehi A. R., "An efficient chaotic water cycle algorithm for optimization tasks," Neural Computing and Applications, vol. 28, no. 1, pp. 57-85, 2017.

[17] Dyck R., Sadiq R., Rodriguez M., Simard S., Tardif R., "A comparison of membership function shapes in a fuzzy-based fugacity model for disinfection byproducts in indoor swimming pools," International Journal of System Assurance Engineering and Management, vol. 8, no. 4, pp. 2051-2063, 2017.

[18] K. Lenin, B. Ravindhranath Reddy, M. Suryakalavathi, "Modified Monkey Optimization Algorithm for Solving Optimal Reactive Power Dispatch Problem," Indonesian Journal of Electrical Engineering and Informatics (IJEEI), Vol. 3, No. 2, pp. 55-62, 2015.

[19] M. Abdel-Baset, I. M. Hezam, "An Effective Hybrid Flower Pollination and Genetic Algorithm for Constrained Optimization Problems," Advanced Engineering Technology and Application an International Journal, vol. 4, pp. $272734,2015$.

[20] I. M. H. Mohamed Abdel-Baset, "An Improved Flower Pollination Algorithm Based on Simulated Annealing for Solving Engineering Optimization Problems," Asian Journal of Mathematics and Computer Research, vol. 3, no. 2, pp. 83-91, 2015.

[21] Saad O. M., Hassan B. M. A., \& Hizam I. M., "Optimizing the underground water confined steady flow using a fuzzy approach,” International Journal of Mathematical Archive, vol. 2, no. 6, pp. 949-957, 2011.

[22] Wijayaningrum V. N., \& Mahmudy W. F., "Optimization of Ship's Route Scheduling Using Genetic Algorithm," Indonesian Journal of Electrical Engineering and Computer Science, vol. 2, no. 1, pp. 180-186, 2016.

[23] Shireen S. Sadiq, Adnan Mohsin Abdulazeez, Habibollah Haron, (2020) "Solving multi-objective master production schedule problem using memetic algorithm," Indonesian Journal of Electrical Engineering and Computer Science, vol. 18, no. 2, pp. 938-945, 2020.

[24] E. M. Badr and M. I. Moussa, "An Upper Bound of Radio k-coloring Problem and its Integer Linear Programming Model," Wireless and Networks, 2019.

[25] Badr E. M. and Aloufi K., "A Robot's Response Acceleration Using the Metric Dimension Problem," Preprints, 2019.

[26] Badr E. M., Almotairi S., "On a Dual Direct Cosine Simplex Type Algorithm and Its Computational Behavior," Mathematical Problems in Engineering, (accepted), 2020. 


\section{BIOGRAPHIES OF AUTHORS}

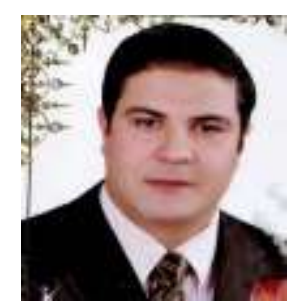

Elsayed Badr is an Associate professor of computer science at Benha Faculty of Computers \& Informatics; Benha University in Egypt. He received his Ph.D. degree in Parallel Algorithms (mainly in parallel graph algorithms) in 2006 from the University of Macedonia; Greece. Dr. Badr holds a Certificate of Quality Assurance from the university of Benha, Egypt and M.Sc. in graph theory and graph algorithms applications, B.Sc. in Mathematics from Benha faculty of science in Egypt. In addition to over 8 years of teaching and academic experiences In Egypt and Greece, Dr. Badr has accumulated broad practical experiences and developed a solid set of skills in algorithms, graph labeling, Wireless Networks, Distributed System, Parallel Programming and linear programming

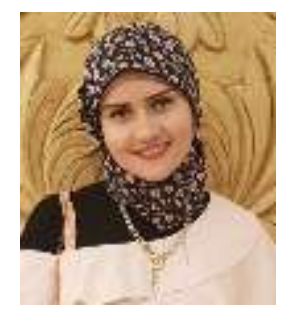

Horia Elgendy is Demonstrator in Engineering Mathematics at the Faculty of Engineering in Benha. Banha University in Egypt. She received her a bachelor's degree in electrical engineering specializing in control and power engineering in 2014 from the Benha Faculty of Engineering, Benha University. In addition to more than 4 years of educational and academic experience in Egypt. 